\title{
Interlayer vacancy diffusion and coalescence in graphite
}

\author{
T. Trevethan, C. D. Latham, M. I. Heggie, and M. J. Rayson \\ Department of Chemistry, University of Surrey, Guildford, Surrey GU2 7XH, United Kingdom \\ P. R. Briddon \\ School of Electrical and Electronic Engineering, University of Newcastle upon Tyne, Newcastle NE1 7RU, United Kingdom
}

(Received 23 May 2014; revised manuscript received 1 September 2014; published 13 November 2014)

\begin{abstract}
Due to the layered nature of graphite, the migration and interaction of point defects in the graphite crystal structure are highly anisotropic, and it is usually assumed that individual mobile lattice vacancies are confined to diffuse on a single plane. We present the results of $a b$ initio calculations based on density functional theory which demonstrate that vacancies can, in fact, move between adjacent planes when they interact with one another via relatively low energy pathways, often with barriers of less than $1 \mathrm{eV}$. These interlayer transition mechanisms can significantly alter both the kinetics of point-defect aggregation and coalescence and also the resultant morphologies of multivacancy complexes that form as a result of migrating vacancies interacting.
\end{abstract}

DOI: 10.1103/PhysRevB.90.174108

PACS number(s): 61.72.Bb, 61.72.up, 61.72.Lk, 61.80.Hg

\section{INTRODUCTION}

Graphite and related structures such as multilayer graphene remain important materials in myriad applications. One of the most critical of these is in the use of graphite as a neutron moderator and reflector in the cores of both currently operating and planned next-generation fission reactors, where the structural integrity of the material must be guaranteed for decades in inaccessible and extremely harsh environments. Irradiation with fast neutrons, as well as heavy ions and electrons, results in macroscopic property changes to graphite components, most notably dimensional change (swelling in the direction perpendicular to the planes and contraction parallel to them) $[1,2]$ but also changes to elastic properties, thermal expansion, and electrical conductivity [3-6].

The underlying atomistic mechanisms driving these property changes are still not fully understood and are the subject of much debate even after many years of intensive research. It is currently accepted that energetic particles (e.g., fast neutrons) initiate collision cascades within the crystal structure that eventually result in a distribution of point defects (Frenkel pairs) comprising lattice vacancies and interstitial atoms [7-9]. This population of point defects may then evolve via thermal activation, and individual defects will interact and react with each other, either annihilating (pairs of vacancies and interstitials) or coalescing to form complexes, extended defects, and dislocations, which will in turn lead to the observed material property changes [7]. Understanding how lattice vacancies migrate and coalesce is therefore crucial to developing a full atomistic model of the response of graphite to irradiation. An important feature of this process is to determine the structures (or morphologies) of the complexes adopted by coalescing vacancies. Coalescence into extended vacancy lines, essentially a line of missing atoms within the graphene sheets from which graphite is composed [7,10,11], will lead to a contraction of the sheet when this "slot" exceeds a critical length and it closes up or heals [12]. Coalescence into "loops" or holes will not lead to any in-plane contraction and instead will form disks of missing material.
A variety of experimental evidence can shed light on the morphologies that will result from vacancy coalescence in irradiation-damaged graphite, including both high-resolution transmission electron microscopy (HRTEM) images and positron annihilation measurements. Resolution in HRTEM images of bulk graphite is limited to the observation of prismatic edges and a cross section of the topology of the individual layers. However, there is evidence for the formation of vacancy loops (holes), as well as extended defects linking adjacent planes as a result of electron irradiation [13,14]. Large prismatic vacancy loops have been observed to form in neutronirradiated graphite at temperatures above $900^{\circ} \mathrm{C}$ [15-17]; however, very small loops (six or fewer vacancies) would not have been visible in these experiments [11]. In bulk graphite samples, it is not possible to resolve the positions of individual atoms with HRTEM [18], so the existence of in-plane healed vacancy lines would not be seen. Positron annihilation experiments have shown evidence of small vacancy loops forming in the graphite structure as a result of irradiation at lower temperatures [19,20], indicating the presence of $V_{4}$ and $V_{6}$ loops (voids) resulting from fast neutron irradiation. Again, this method would not detect the presence of healed lines in either crystallographic direction due to the lack of any void.

These observations contrast with recent aberrationcorrected HRTEM images of single-layer suspended graphene in which the structure of the lattice can be seen clearly with atomic resolution [21]. These images do reveal morphologies that correspond to the formation of vacancy lines running in the two primary crystallographic directions, with "healing" across the lines to form dislocations, as well as other structures involving Stone-Wales-type bond rotations [22-24]. However, they do not show evidence of the regular formation of the small vacancy loops (or holes) of the same type inferred from the positron annihilation experiments (specifically, the $V_{4}$ and $V_{6}$ loops) [21]. These results suggest that there may be a fundamental difference in the way that vacancies coalesce in single-layer graphene compared with the multilayered graphite structure, which seems to contradict the current understanding that vacancies only diffuse within a single plane and that 
single-layer graphene is a good model system to understand the behavior and coalescence of vacancies in bulk graphite. Several previous investigations of the energetics of multivacancy defects in graphene layers with ab initio modeling have found that the loop morphologies are low-energy structures, at least for the small loops $\left(V_{4}\right.$ and $\left.V_{6}\right)$ [25,26]. Other configurations, involving multiple bond rotations, can be even lower in energy [27]. The morphologies that actually form in a particular system will depend on the mechanism of formation and the kinetically accessible pathways to coalescence at a particular temperature.

In this work we present the results of first-principles density functional theory (DFT) calculations which demonstrate that, contrary to commonly held assumptions about defect behavior in graphite, vacancies can migrate between adjacent planes during coalescence via barriers as low as $0.9 \mathrm{eV}$ and that this fundamentally changes both the mechanisms by which vacancies coalesce and the morphologies of the multivacancy complexes that can result. This behavior arises due to the way individual vacancies can form stable interlayer complexes when undercoordinated atoms surrounding a vacancy defect form chemical bonds between adjacent planes. This occurs whenever vacancies in neighboring planes are in specific registries that are close together, where the vacancies may bond in several possible ways. In the first instance, interlayer divacancy complexes form. These complexes can either act as intermediate states for the formation of the more stable coplanar divacancy (the 5-8-5 structure) via the diffusion of a vacancy between the layers or can nucleate the formation of larger interlayer complexes (ramps). The larger complexes subsequently evolve in two ways. Either they grow further into extended defects linking neighboring layers, or interlayer diffusion produces vacancy loops that are not kinetically accessible from coplanar aggregation alone.
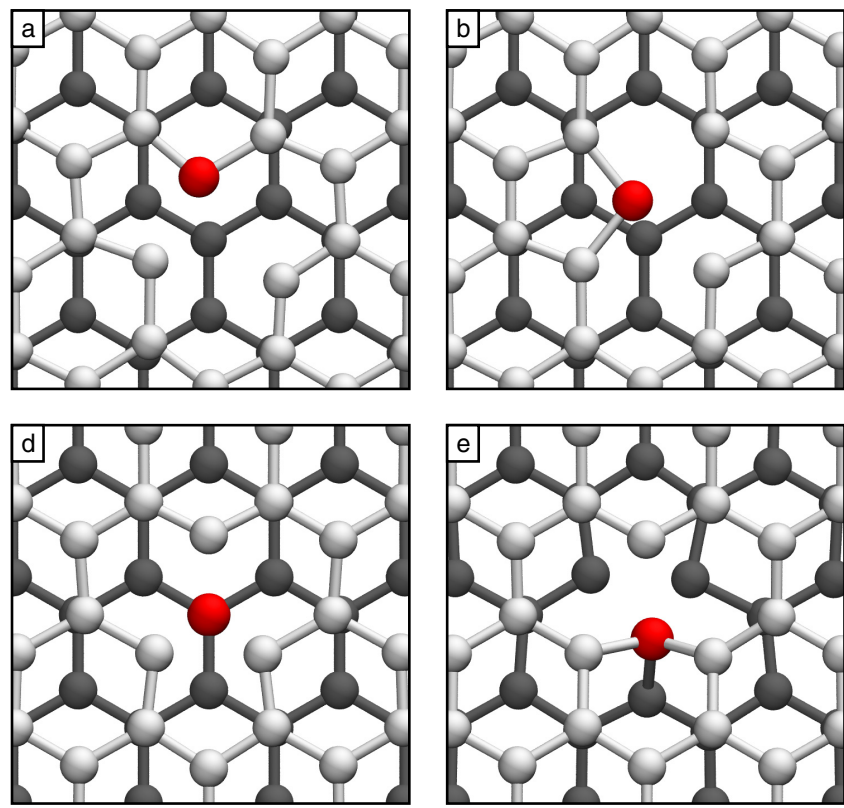

\section{METHODOLOGY}

To investigate the coalescence of vacancies in the graphite structure with both accuracy and computational efficiency, we employ the DFT method as implemented in the AIMPRO simulation package [28-31]. The methodology is similar to that described in previous work $[32,33]$. Calculations are performed with both the local-density approximation (LDA) [34] and the PBE96 generalized gradient approximation (GGA) [35] by Perdew, Burke, and Ernzerhof for the exchange-correlation functional. Norm-conserving pseudopotentials are used in place of core electrons [36]. Values for energies are given first for the LDA, followed by the GGA values in parentheses. Neither of these functionals correctly describes dispersion interactions, which are an important component of the interlayer binding in perfect graphite; however, the LDA does correctly reproduce the interlayer interaction energy and interlayer separation (whereas the GGA predicts no interlayer binding) [33]. We find that relative energy changes and barriers are usually insensitive to the exchange-correlation functional employed, which suggests that the results are not significantly affected by the lack of dispersion.

Model defects are constructed in orthorhombic supercells containing either two or four graphene layers, where each layer has 72 atoms (144 or 288 atoms in total) when no defect is present to guarantee convergence with system size. A Monkhorst-Pack $\boldsymbol{k}$-point mesh of $4 \times 4 \times 2$ points was determined to provide total energy convergence $\epsilon \lesssim$ $0.1 \mathrm{meV} /$ atom. Optimized structures are determined by minimizing the forces on all free atoms, and the total energy of the system is determined with a conjugate-gradient algorithm. Activation energy barriers and minimum-energy pathways are calculated using the climbing-image nudged elastic band (NEB) method [37,38], with up to 15 images in each chain.
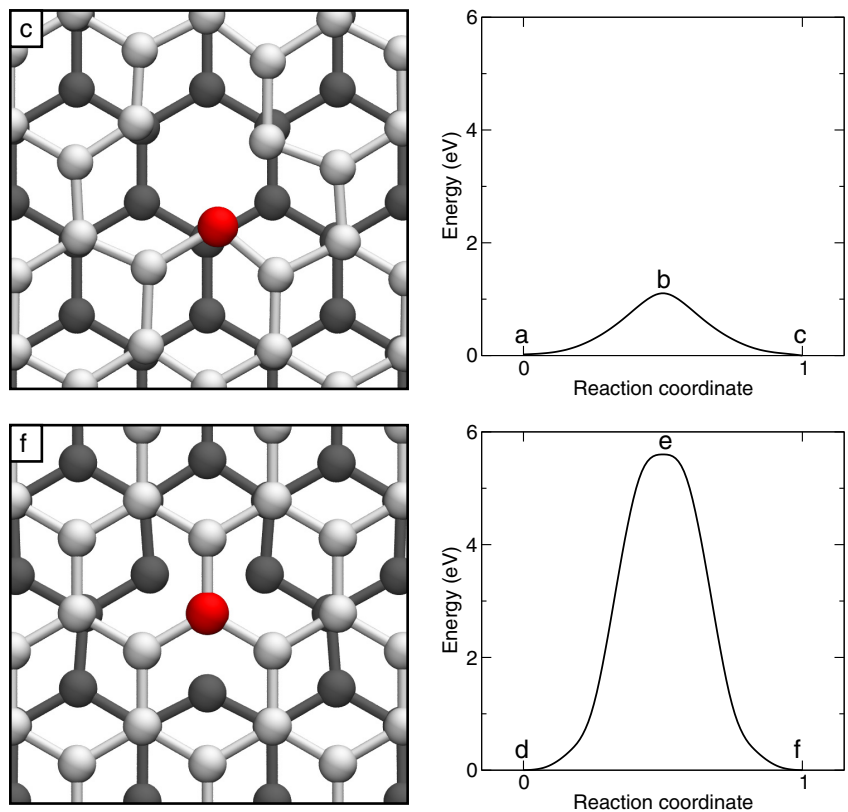

FIG. 1. (Color online) Monovacancy diffusion. (a) Initial structure, (b) saddle point structure, and (c) final structure of the in-plane transition. (d) Initial structure, (e) saddle point structure, and (f) final structure of the transition of an $\alpha$ monovacancy between planes. The upper-layer atoms are white, lower-layer atoms are dark gray, and the moving atom is red. The minimum energy paths for each transition are shown on the right. 


\section{RESULTS}

The monovacancy in graphite has a formation energy of $8.0 \mathrm{eV}$ (7.4 eV GGA) [33]. Upon formation, the vacancy undergoes a spontaneous Jahn-Teller distortion [39] in which a pairwise reconstruction occurs, weakly binding two of the undercoordinated atoms, leaving one dangling bond [40,41]. This asymmetric reconstruction can rotate to the other two equivalent orientations via a small $(\sim 0.1 \mathrm{eV})$ barrier [40]. The predicted activation barrier for migration of a monovacancy within the layer is $E_{a} \approx 1.1 \mathrm{eV}$ [33], which means that the process will be thermally activated above about $100{ }^{\circ} \mathrm{C}$. Direct observations of vacancies on the surfaces of graphite crystals using scanning tunneling microscopy appear to show that $E_{a} \approx 1 \mathrm{eV}$ [42]. The migration process for a monovacancy between adjacent lattice sites, including the structure of the saddle point, is illustrated in Figs. 1(a)-1(c). In order for the monovacancy to migrate to an adjacent plane, it must exchange places with a carbon atom across the $3.35-\AA$ gap that separates them, which leads to a much larger barrier. There are two pathways for this to occur: one where a vacancy located at an $\alpha$ site moves to the nearest-neighbor $\alpha$ site in the adjacent plane and one where a vacancy located at a $\beta$ site moves to the closest $\alpha$ site in the adjacent plane. The first of these, which is depicted in Figs. 1 (d)-1(f) along with the saddle point configuration, has a barrier $E_{a} \approx 5.6 \mathrm{eV}$, while the second has a barrier $E_{a} \approx 6.5 \mathrm{eV}$. Both of these barriers are very high and would not be thermally accessible except at extremely high temperatures (above $2000 \mathrm{~K}$ ). The lower of these two values matches very well with the activation energy for interlayer vacancy diffusion inferred from experimental observations of the annealing of interstitial loops in irradiated graphite (approximately $5.4 \mathrm{eV}$ ) [43,44].

This potential energy surface effectively confines isolated monovacancies so that they diffuse within a single layer
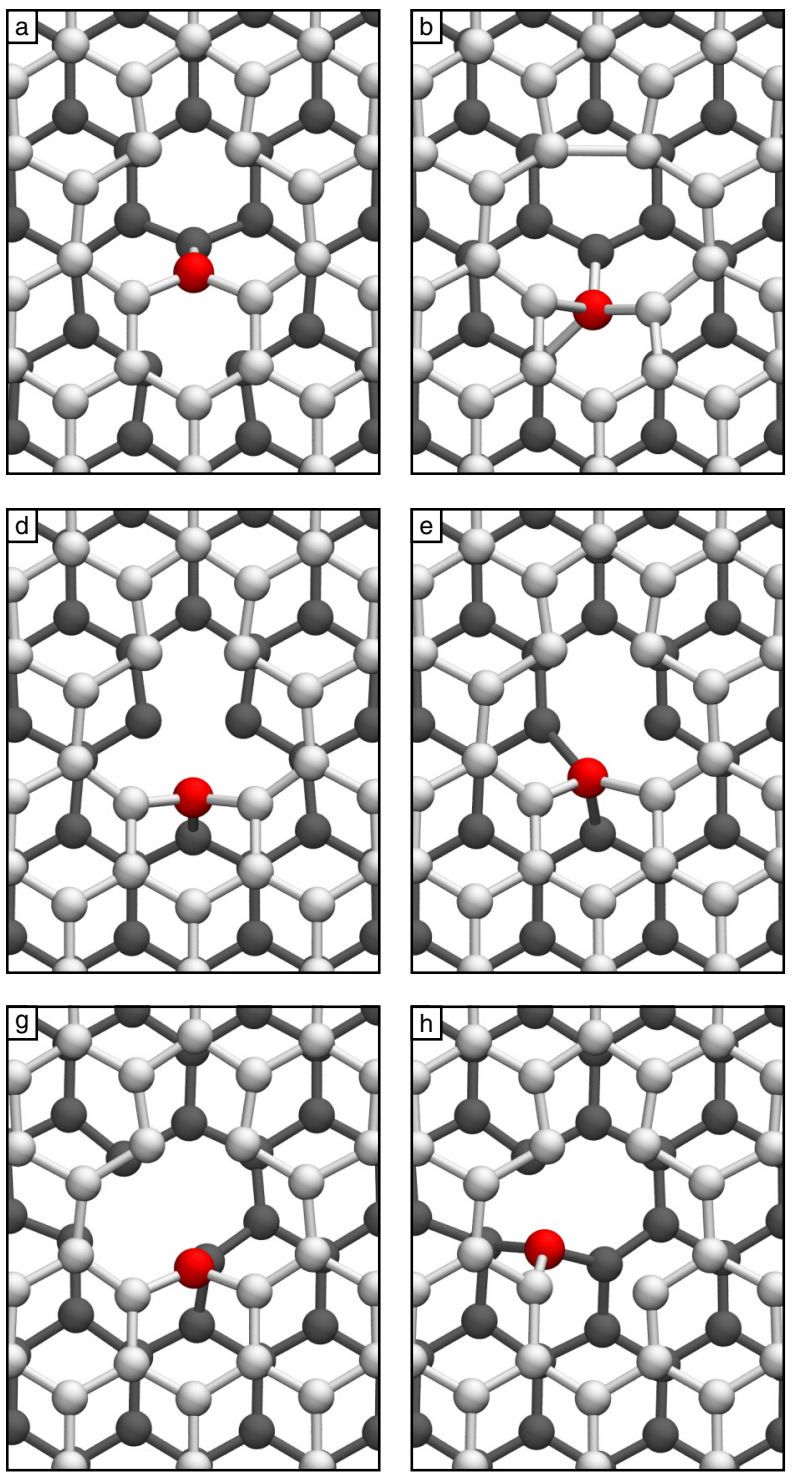
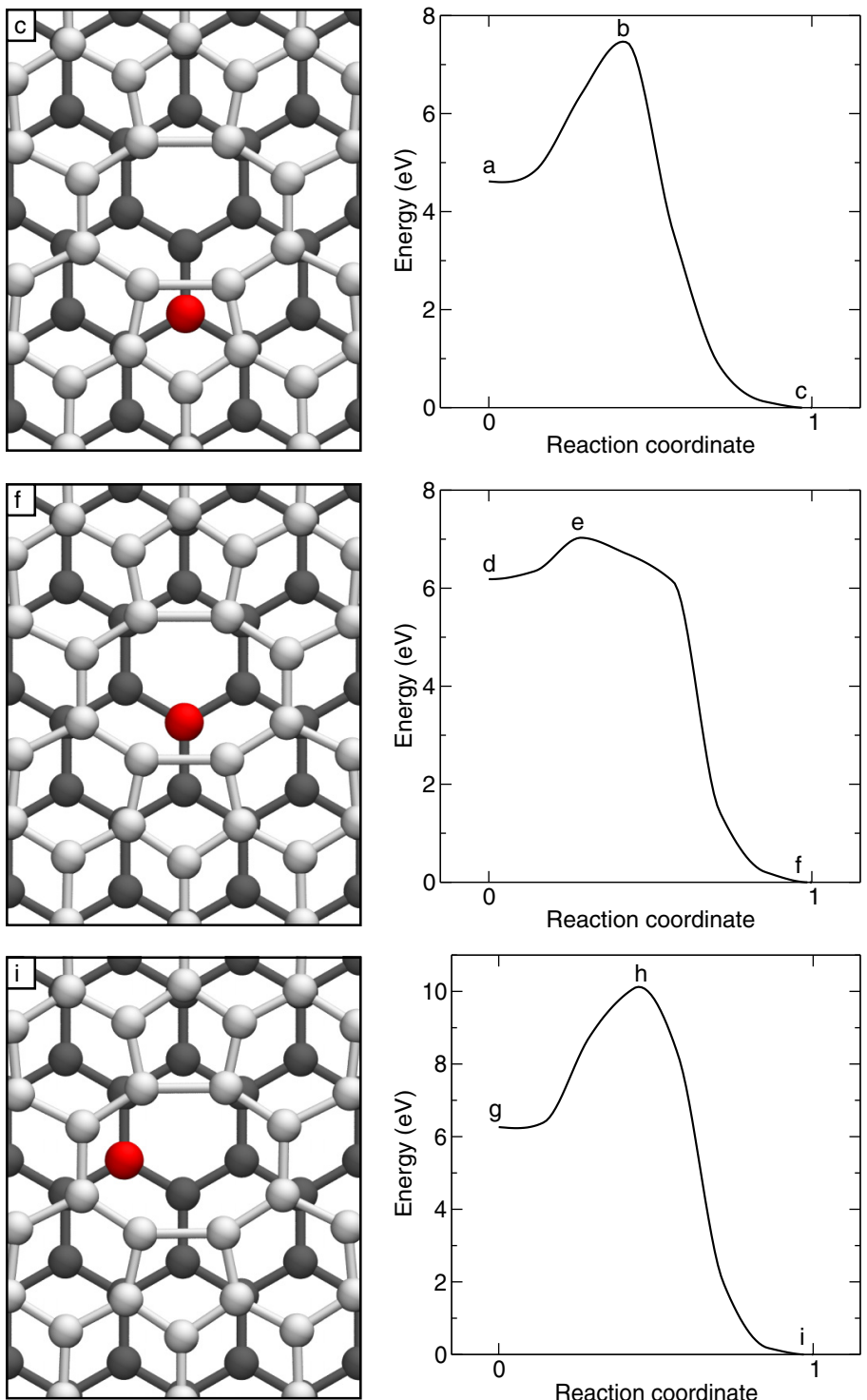

FIG. 2. (Color online) Interlayer divacancy coalescence to the in-plane 5-8-5 structure. (a) The $V_{2,1}^{\beta \beta *}$ structure, (b) saddle point structure, and (c) final structure. (d) The $V_{2,1}^{\alpha \beta}$ structure, (e) saddle point structure, and (f) final structure. (g) The $V_{\beta \beta}^{2,1}$ structure, (h) saddle point structure, and (i) final structure. The atom that moves between the layers is red. The minimum energy paths for each transition are shown on the right. 
TABLE I. Binding energies $E_{b}$, interlayer bond formation activation energies $E_{a}^{b}$, and activation energies for interlayer vacancy diffusion $E_{a}^{c}$ for the three interlayer divacancy structures shown in Fig. 2.

\begin{tabular}{|c|c|c|c|c|c|c|}
\hline & \multicolumn{2}{|c|}{$V_{2,1}^{\beta \beta *}$} & \multicolumn{2}{|c|}{$V_{2,1}^{\alpha \beta}$} & \multicolumn{2}{|c|}{$V_{\beta \beta}^{2,1}$} \\
\hline & LDA & GGA & LDA & GGA & LDA & GGA \\
\hline$E_{b}$ & 3.3 & 3.0 & 1.5 & 1.2 & 1.8 & 1.4 \\
\hline$E_{a}^{b}$ & 0.3 & 0.3 & 0.3 & 0.3 & 0.2 & 0.3 \\
\hline$E_{a}^{c}$ & 2.8 & 2.8 & 0.9 & 0.8 & 3.9 & 3.8 \\
\hline
\end{tabular}

(at temperatures below $2000 \mathrm{~K}$ ). When two diffusing monovacancies meet within the same plane, they will coalesce to form the stable, immobile, and widely observed 5-8-5 divacancy structure $[21,23,45]$, following the reaction pathway determined in Ref. [33] and releasing $8.0 \mathrm{eV}(7.7 \mathrm{eV})$ in the process. As first proposed in Ref. [9] and analyzed in detail in Ref. [33], when two mobile monovacancies in adjacent layers come into registry in certain configurations, undercoordinated atoms surrounding the vacancy in each layer can create an $s p^{2}$ bond across the interlayer gap that releases a significant amount of energy. The three configurations in which these complexes can form in $\mathrm{AB}$ graphite are shown in Figs. 2(a), 2(d), and 2(g), and the energy release $E_{b}$ for the formation of each structure from the two separated vacancies is given in Table I. For each of these complexes, a small activation energy is needed to form the interlayer bond when the vacancies are in registry $\left(E_{a}^{b}\right)$, which we have calculated using the NEB method; the values are given in Table I. The largest of these activation energies is only $0.3 \mathrm{eV}$, so these complexes will be formed almost instantaneously at temperatures where monovacancies are mobile, as soon as they diffuse into registry with each other. The notation for labeling the interlayer complexes is taken from Ref. [33]: the subscript denotes, first, the number of vacancies in the complex and, second, the number of interlayer bonds. The superscript denotes the lattice position of each vacancy $(\alpha$ or $\beta$ ), with an asterisk indicating the next-nearest neighbor.

Once they have formed, these complexes are relatively stable with respect to dissociation: the barrier to break the interlayer bond is the sum of the coalescence energy and the activation energy to coalescence; thus, these complexes are unlikely to dissociate below about $500 \mathrm{~K}$. However, in addition to disassociating, both of the atoms forming the interlayer bond can move into one of the two layers, leaving a coplanar divacancy structure in one layer. This process removes a vacancy from the adjacent layer (as shown for each type of complex in Fig. 2). Thus, the net effect is that a vacancy moves from one layer to the next. In each case this transition will result in a substantial energy release due to the relative stability of the 5-8-5 coplanar divacancy structure. This energy release is equal to the difference between the binding energies of the coplanar divacancy defect (LDA: $8.0 \mathrm{eV}$, GGA: 7.7 eV) and the interalyer complex $\left(E_{b}\right)$.

The calculated binding and activation energies for crosslayer migration are given in Table I, and the minimum energy paths from the NEB calculations (splined) are shown on the right side of Fig. 2 for each structure. Since the barrier for

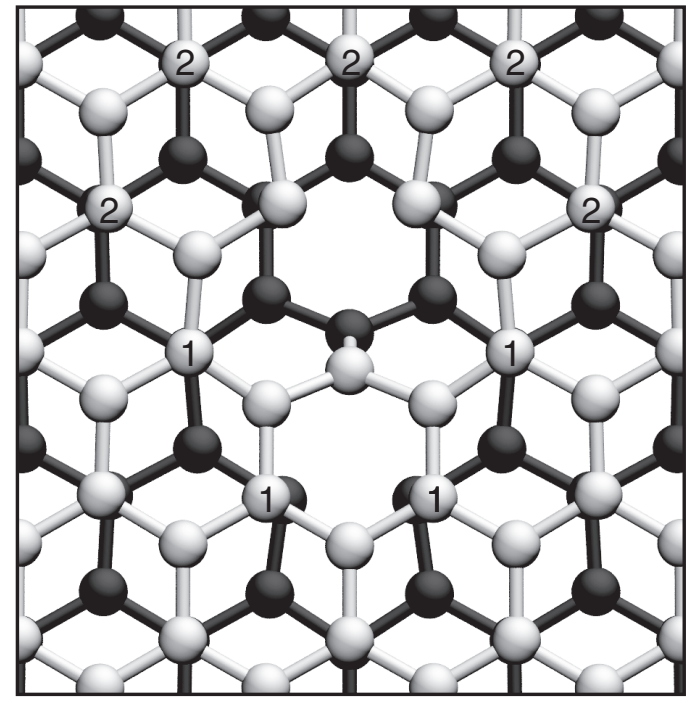

FIG. 3. (a) The $V_{2,1}^{\beta \beta *}$ structure with the third-nearest-neighbor lattice positions to the vacancy in the upper layer (labeled 1 or 2).

the $V_{2,1}^{\alpha \beta}$ complex to collapse is only $0.9 \mathrm{eV}(0.8 \mathrm{eV})$, it will convert almost immediately into a coplanar nearest-neighbor divacancy at temperatures where vacancies are mobile. Both $V^{\beta \beta}$ complexes have barriers to transform into a coplanar divacancy that are significantly larger (nearly $3 \mathrm{eV}$ or more), which, with their high barriers to dissociation, means that in isolation they are stable up to temperatures well above the onset of vacancy mobility. The collapse of the $V_{\beta \beta}^{2,1}$ structure to the coplanar divacancy was simulated for bilayer graphene in Ref. [46] in which a barrier of $3 \mathrm{eV}$ was found, which is comparable to but slightly less than the value of $3.8 \mathrm{eV}$ we find for the same transition.

The situation for the two stable $V^{\beta \beta}$ complexes changes with the addition of another mobile vacancy. Both structures can transform to either the ground-state coplanar trivacancy $\left(V_{3}\right)$ structure or an interlayer $V_{3}$ structure, depending on the trajectory of the additional monovacancy, i.e., the lattice position it diffuses to relative to the interlayer divacancy structure. A schematic of the $V_{2,1}^{\beta \beta *}$ structure is shown in Fig. 3, with the third-nearest-neighbor lattice positions surrounding the vacancy labeled 1 or 2 . If an additional mobile vacancy migrates to any of the positions labeled 1, then the structure shown in Fig. 4(b) will result from a transition over a small barrier $(<0.3-0.4 \mathrm{eV})$. An example is shown in Fig. 4(a). From this structure [Fig. 4(b)] the two-coordinated atom sitting between the layers can then move into the lower layer, resulting in a $V_{3}$ coplanar complex in the upper layer (which is the ground state) via a $0.8 \mathrm{eV}$ barrier and releasing $6.2 \mathrm{eV}$ in the process [Fig. 3(d)].

The potential energy surface for an additional vacancy diffusing to the other stable $V_{2,1}^{\beta \beta}$ structure is very similar, and a monovacancy accessing the same positions labeled 1 in Fig. 3 relative to the interlayer bond will result in a two-coordinated atom sitting between the layers. From this configuration, as before, the two-coordinated atom can then move into the lower layer, leaving the $V_{3}$ coplanar complex in the upper layer. The activation barrier $(0.5 \mathrm{eV})$ for this transition is very similar to the monovacancy coalescence with the $V_{2,1}^{\beta \beta *}$ structure. 

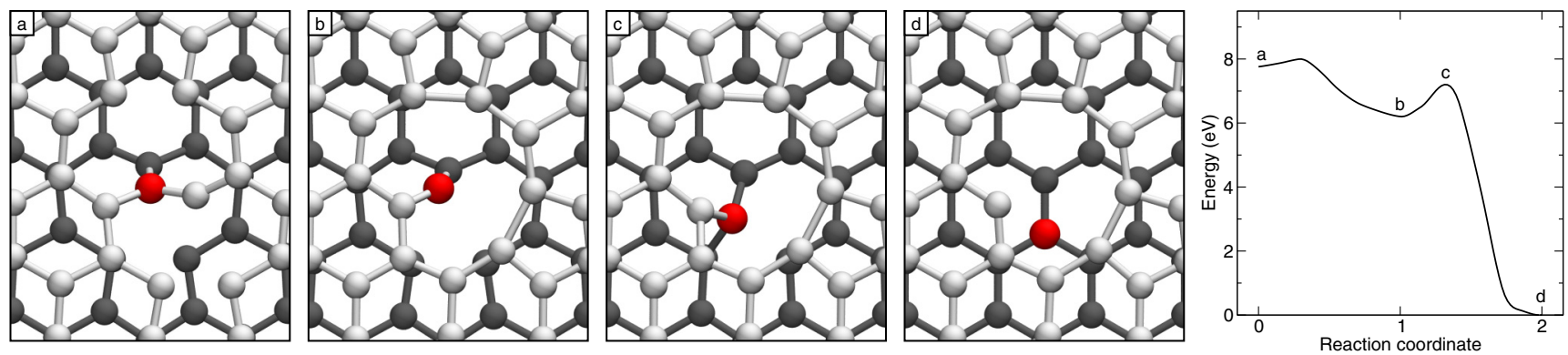

FIG. 4. (Color online) Coalescence of monovacancy with the $V_{2,1}^{\beta \beta *}$ structure. (a) A monovacancy migrated to a third-nearest-neighbor position labeled with a 1. (b) The resulting structure after a $0.2 \mathrm{eV}$ barrier transition, (c) the saddle point structure for interlayer migration, and (d) the final coplanar trivacancy structure.

The coplanar $V_{3}$ complex can therefore be formed from both the coplanar [10] and interplane coalescence of monovacancies, as can the 5-8-5 divacancy. This structure, which

is the lowest-energy $V_{3}$ morphology [10], comprises two reconstructed fivefold rings and a tenfold ring, with a single undercoordinated atom providing a dangling bond.
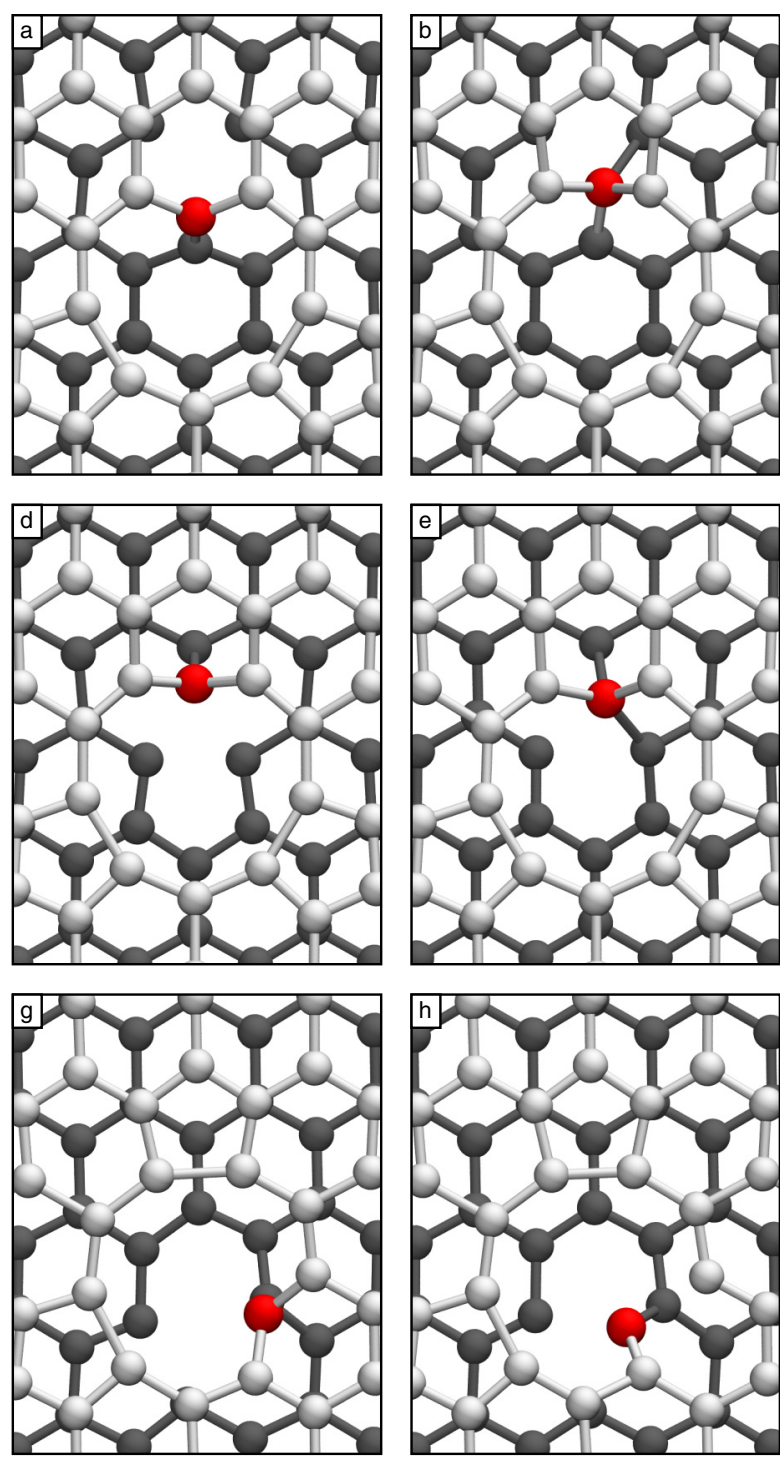
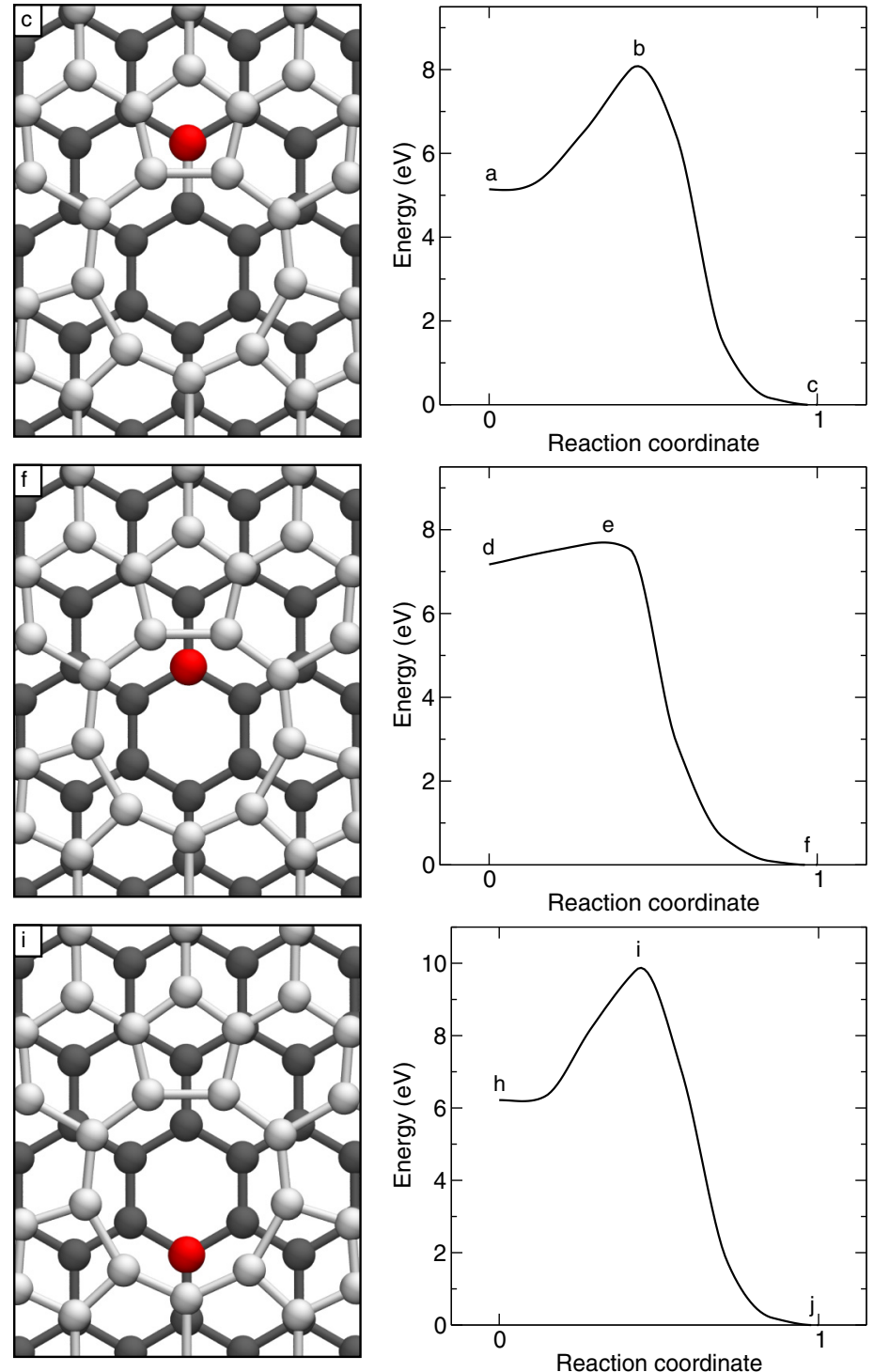

FIG. 5. (Color online) Interlayer coalescence of a monovacancy with a coplanar trivacancy. (a), (d), and (g) The three configurations of a monovacancy bound to a trivacancy. (b), (e), and (h) The corresponding saddle points for interlayer migration. (c), (f), and (i) The fully coalesced coplanar quad vacancies. The atom that moves between the layers is red. The corresponding minimum energy paths for each transition are shown on the right. 
TABLE II. Interlayer binding energies $E_{b}$, activation energies for interlayer vacancy motion $E_{a}$, and energy releases $E_{c}$ for the three interlayer structures (a, d, g) shown in Fig. 5.

\begin{tabular}{|c|c|c|c|c|c|c|}
\hline & \multicolumn{2}{|c|}{$\mathrm{a}$} & \multicolumn{2}{|c|}{$\mathrm{d}$} & \multicolumn{2}{|c|}{ g } \\
\hline & LDA & GGA & LDA & GGA & LDA & GGA \\
\hline$E_{b}$ & 3.2 & 3.2 & 1.4 & 1.2 & 2.3 & 2.1 \\
\hline$E_{a}^{c}$ & 3.0 & 2.9 & 0.4 & 0.5 & 3.6 & 3.6 \\
\hline$E_{c}$ & 5.0 & 5.1 & 7.0 & 7.2 & 6.1 & 6.2 \\
\hline
\end{tabular}

This dangling bond can bind to and immobilize diffusing monovacancies in adjacent planes when they come into the same registry positions that result in the formation of the interlayer divacancies. The three structures that can form are shown in Figs. 5 (a), 5(d), and 5(g). The formation of these structures releases energies similar to those of the formation of the interlayer divacancies of the same motif, which are given in Table II. For each of these structures, there is no barrier to the formation of the interlayer bond, which is most likely due to the increased softness or flexibility of the undercoordinated atom of the $V_{3}$. As in the case of each of the interlayer divacancies, there is a transition pathway for the $\alpha$ atom forming the interlayer bond from the $V_{3}$ to move into the monovacancy position in the adjacent layer, extending the $V_{3}$ to a $V_{4}$ loop and restoring the perfect lattice in the adjacent layer (Fig. 5). This process is also accompanied by a substantial release of energy due to the high stability of the $V_{4}$ loop. The calculated energy release and barriers for each of these transitions are given in Table II. As before, the barrier for the interlayer motion of the monovacancy is rather high for the two $V^{\beta \beta}$-type structures. However, for the $V^{\alpha \beta}$-type complex, where the $V_{3}$ undercoordinated atom is directly in line with the monovacancy position on an $\alpha$ site, this barrier is relatively small, only $0.4 \mathrm{eV}$. The other two structures are relatively stable and can act to nucleate the formation of more complex defects or can collapse into coplanar loops as additional mobile vacancies coalesce with them.

Returning to the coalescence of additional monovacancies to the stable $V^{\beta \beta}$ interlayer divacancies: if an additional mobile vacancy diffuses to any of the positions labeled 2 in Fig. 3, the interlayer bond will remain. This will result in a single vacancy in one layer bonded to a 5-8-5 divacancy in the adjacent layer, but with one of the fivefold rings broken to form the interlayer bond. This is a structure which could also form from the coalescence of a single mobile vacancy with an immobile 5-8-5 divacancy in an adjacent layer. Additional monovacancies can then coalesce with this complex in either layer. The coalescence of an additional three monovacancies, two in the initial monovacancy layer and one in the initial divacancy layer, making six vacancies in total, can result in the structure shown in Fig. 5(a). This structure comprises two coplanar $V_{3}$ complexes in each layer that face each other, with the overlapping edge $\alpha$ atoms forming two bonds between the layers [14]. From this bound interlayer state, three of the atoms in one layer, forming half of the central hexagonal unit (highlighted in red), can move into the adjacent layer in a single concerted transition, releasing $4.4 \mathrm{eV}$ and resulting in the formation of a $V_{6}$ ring in one layer. The structure is illustrated in Fig. 6(d). The activation barrier for this process is surprisingly low, only $0.7 \mathrm{eV}$, which would be crossed as soon as the complex is formed. During the course of migrating from one layer to the neighboring one, the moving atoms maintain bonds with both layers, allowing the system to reorganize its structure while barely breaking any bonds.

Achieving this low activation barrier and high-energy release for the simultaneous interlayer motion of several atoms requires that the interlayer movement results in a very stable coplanar vacancy loop in one layer while the other layer is restored to the perfect lattice. In the previous example, this means the $V_{3}$ defects in each layer must be exactly in registry. If the coalescence happened in a different sequence, then the barrier to interlayer vacancy motion would be much higher due to the stabilizing effect of a fivefold ring. For example, if two monovacancies coalesced to the divacancy before an additional two monovacancies coalesced to the bound monovacancy in the adjacent layer, then the structure shown in Fig. 7(a) would result (i.e., a $V_{4}$ line or loop in one layer and a $V_{3}$ in the other, connected with two bonds). This one additional vacancy dramatically stabilizes the interlayer ramp defect. In the transition to the structure shown in Fig. 7(b), the system releases $4.6 \mathrm{eV}$; however, the barrier to form this in-plane $V_{7}$ loop is now $3.4 \mathrm{eV}$. An alternative structural change is shown in Fig. 7(c), where the $V_{6}$ loop is formed in one layer and leaves a monovacancy in the other: this transition is not energetically favorable, and the final structure is $2.5 \mathrm{eV}$ higher in energy, with a barrier of $2.9 \mathrm{eV}$ to form. However, the reverse of this transition demonstrates that there is only a barrier of $0.4 \mathrm{eV}$ to the exothermic formation of a ramp defect [i.e., Fig. 7(a)]
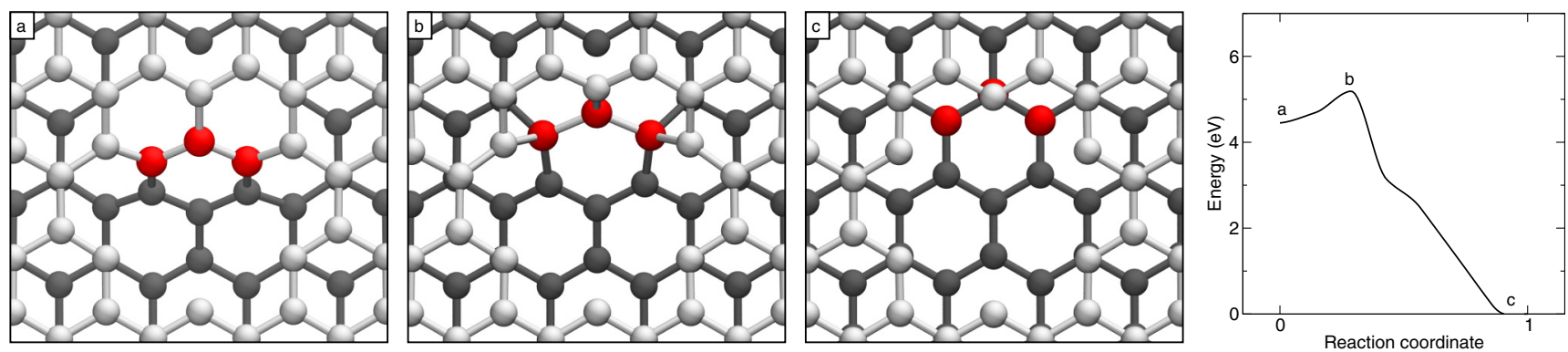

FIG. 6. (Color online) (a) The structure of an interlayer $V_{3}-V_{3}$ complex. (b) The saddle point structure for the collective interlayer migration of the $V_{3}$. (c) The coplanar $V_{6}$ loop formed from the movement of the three highlighted (red) atoms between the layers. The minimum energy path is shown on the right. 

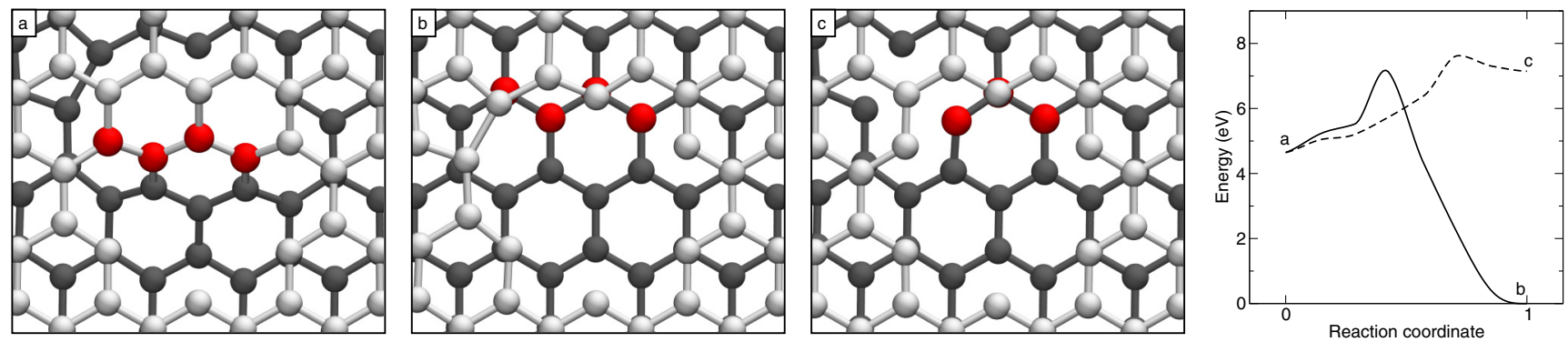

FIG. 7. (Color online) (a) An interlayer $V_{4}-V_{3}$ complex. (b) An in plane $V_{7}$ loop formed from the movement of the four highlighted (red) atoms between the layers. (c) An in-plane $V_{6}$ loop in one layer and a monovacancy in the other layer formed from the movement of three of the four highlighted atoms between the layers. The corresponding minimum energy paths for each transition are shown on the right.

from the interaction of a monovacancy in one layer with a $V_{6}$ loop in an adjacent layer.

An interlayer ramp defect of a different morphology can also form from the coalescence of additional mobile vacancies to the interlayer $V_{4}$ structures shown in Fig. 3. Here, further interlayer diffusion of atoms will be prevented owing to the stabilizing nature of the reconstructed fivefold rings, resulting in the accumulation of additional vacancies.

\section{DISCUSSION AND CONCLUSION}

The DFT-based simulations presented in this work demonstrate the various ways in which vacancies can move between adjacent layers in graphitic structures when they interact and coalesce. This suggests that there should be a fundamental change in our ideas about the behavior of vacancies and their diffusion and aggregation in bulk graphite, multilayer graphene, and multiwalled carbon nanotubes. According to the calculations, interlayer diffusion processes can change both the kinetics of monovacancy aggregation and also the kinetically accessible morphologies of multivacancy complexes.

We have shown low-energy pathways to the formation of both $V_{4}$ and $V_{6}$ coplanar vacancy loops (or holes) that progress via the interlayer movement of atoms (and vacancies). Experimental evidence, in the form of atomic resolution HRTEM images of single-layer graphene, suggests that these multivacancy morphologies are not routinely observed in this material [21], and recent modeling has also suggested that they are kinetically inaccessible from purely in-plane aggregation of mobile monovacancies in graphene [10]. There is experimental evidence for their existence, however, in bulk irradiated graphite $[13,15-17,20]$, and our results provide a possible explanation for this discrepancy. When vacancies can interact through adjacent planes, new pathways are opened for vacancy coalescence. The specific processes presented and discussed here deal with only relatively small aggregates. As the number of vacancies is increased, different and more complex interplane aggregate structures and transitions are possible, and we suggest that one possibility is the formation of extended defects that connect neighboring graphene layers in graphite together via a sheet or strip of graphene, similar to a ramp between the floors in a multistory car park [14].

The types of structures and transitions described here are, of course, only part of the overall picture for the microstructural evolution of a population of point defects created as a consequence of irradiation in bulk graphite, and these mechanisms will be competing against other processes such as interstitial-vacancy recombination and the purely coplanar coalescence of vacancies. We should therefore expect a mixture of both in-plane and intraplane aggregation mechanisms in the coalescence of vacancies; however, quantitatively assessing the relative contributions of each one will require a full dynamical model incorporating all possible processes for different vacancy populations. Nevertheless, we expect that the processes described here will contribute significantly to the complete understanding of the response of graphite and other graphitic structures to energetic particle irradiation.

\section{ACKNOWLEDGMENTS}

The authors wish to thank the UK EPSRC (Grant No. EP/1003312/1), The Swedish Research Council (Reg. No. 2012-3174), the UK Technology Strategy Board (Project No. 101437), and EDF Energy Nuclear Generation for providing financial support. The views expressed in this work are not necessarily those of the sponsors.
[1] Chemistry and Physics of Carbon, edited by P. L. Walker, Vol. 2 (Arnold, London, 1966).

[2] B. T. Kelly, B. J. Marsden, K. Hall, D. G. Martin, A. Harper, and A. Blanchard, Irradiation Damage in Graphite due to Fast Neutrons in Fission and Fusion Systems, IAEA TECDOC Vol. 1154 (International Atomic Energy Agency, Vienna, 2000).

[3] B. T. Kelly, Physics of Graphite (Applied Science, London, 1981).
[4] M. Burton and T. J. Neubert, J. Appl. Phys. 27, 557 (1956).

[5] T. Iwata, T. Nihira, and H. Matsuo, J. Phys. Soc. Jpn. 36, 123 (1974).

[6] J. H. W. Simmons, Radiation Damage in Graphite, International Series of Monographs in Nuclear Energy Vol. 102 (Pergamon, Oxford, 1965).

[7] R. H. Telling and M. I. Heggie, Philos. Mag. 87, 4797 (2007).

[8] E. Kaxiras and K. C. Pandey, Phys. Rev. Lett. 61, 2693 (1988). 
[9] R. H. Telling, C. P. Ewels, A. A. El-Barbary, and M. I. Heggie, Nat. Mater. 2, 333 (2003).

[10] T. Trevethan, C. D. Latham, M. I. Heggie, P. R. Briddon, and M. J. Rayson, Nanoscale 6, 2978 (2014).

[11] P. A. Thrower, in Chemistry and Physics of Carbon, edited by P. L. Walker, Vol. 5 (Marcel Dekker, New York, 1969), Chap. 3, pp. 217-319.

[12] B. W. Jeong, J. Ihm, and G. D. Lee, Phys. Rev. B 78, 165403 (2008).

[13] C. Karthik, J. Kane, D. P. Butt, W. E. Windes, and R. Ubic, J. Nucl. Mater. 412, 321 (2011).

[14] T. Trevethan, P. Dyulgerova, C. D. Latham, M. I. Heggie, C. R. Seabourne, A. J. Scott, P. R. Briddon, and M. J. Rayson, Phys. Rev. Lett. 111, 095501 (2013).

[15] P. A. Thrower, Carbon 6, 687 (1968).

[16] P. A. Thrower, Br. J. Appl. Phys. 15, 1153 (1964).

[17] P. A. Thrower and R. M. Mayer, Phys. Status Solidi A 47, 11 (1978).

[18] C. R. Seabourne, R. Brydson, M. I. Heggie, C. D. Latham, and A. J. Scott, J. Phys. Conf. Ser. 371, 012061 (2012).

[19] T. Onitsuka, H. Ohkubo, M. Takenaka, N. Tsukuda, and E. Kuramoto, J. Nucl. Mater. 283-287, part 2, 922 (2000).

[20] Z. Tang, M. Hasegawa, T. Shimamura, Y. Nagai, T. Chiba, Y. Kawazoe, M. Takenaka, E. Kuramoto, and T. Iwata, Phys. Rev. Lett. 82, 2532 (1999).

[21] A. W. Robertson and J. H. Warner, Nanoscale 5, 4079 (2013).

[22] F. Banhart, J. Kotakoski, and A. V. Krasheninnikov, ACS Nano 5, 26 (2011).

[23] O. Lehtinen, S. Kurasch, A. V. Krasheninnikov, and U. Kaiser, Nat. Commun. 4, 2098 (2013).

[24] J. H. Warner, E. R. Margine, M. Mukai, A. W. Robertson, F. Giustino, and A. I. Kirkland, Science 337, 209 (2012).

[25] M. Oubal, M. Picaud, M.-T. Rayez, and J.-C. Rayez, Comput. Theor. Chem. 990, 159 (2012)

[26] M. Saito, K. Yamashita, and T. Oda, Jpn. J. Appl. Phys. 46, L1185 (2007).

[27] J. Kotakoski, F. R. Eder, J. C. Meyer, Phys. Rev. B 89, 201406 (2014).
[28] P. R. Briddon and R. Jones, Phys. Status Solidi B 217, 131 (2000).

[29] J. P. Goss, M. J. Shaw, and P. R. Briddon, in Theory of Defects in Semiconductors, edited by D. A. Drabold and S. K. Estreicher, Topics in Applied Physics Vol. 104 (Springer, Berlin, 2007), Chap. 3, pp. 69-94.

[30] M. J. Rayson and P. R. Briddon, Comput. Phys. Commun. 178, 128 (2008).

[31] M. J. Rayson, Phys. Rev. E 76, 026704 (2007).

[32] C. D. Latham, M. I. Heggie, J. A. Gámez, I. Suárez-Martínez, C. P. Ewels, and P. R. Briddon, J. Phys. Condens. Matter 20, 395220 (2008).

[33] C. D. Latham, M. I. Heggie, M. Alatalo, S. Öberg, and P. R. Briddon, J. Phys. Condens. Matter 25, 135403 (2013).

[34] J. P. Perdew and Y. Wang, Phys. Rev. B 45, 13244 (1992).

[35] J. P. Perdew, K. Burke, and M. Ernzerhof, Phys. Rev. Lett. 77, 3865 (1996).

[36] C. Hartwigsen, S. Goedecker, and J. Hutter, Phys. Rev. B 58, 3641 (1998).

[37] G. Henkelman, B. P. Uberuaga, and H. Jónsson, J. Chem. Phys. 113, 9901 (2000).

[38] G. Henkelman and H. Jónsson, J. Chem. Phys. 113, 9978 (2000).

[39] C. A. Coulson, M. A. Herraez, M. Leal, E. Santos, and S. Senent, Proc. R. Soc. London, Ser. A 274, 461 (1963).

[40] A. A. El-Barbary, R. H. Telling, C. P. Ewels, M. I. Heggie, and P. R. Briddon, Phys. Rev. B 68, 144107 (2003).

[41] A. W. Robertson, B. Montanari, K. He, C. S. Allen, Y. A. Wu, N. M. Harrison, A. I. Kirkland, and J. H. Warner, ACS Nano 7, 4495 (2013).

[42] J. I. Paredes, P. Solís-Fernández, A. Martínez-Alonso, and J. M. D. Tascón, J. Phys. Chem. C 113, 10249 (2009).

[43] W. N. Reynolds and P. A. Thrower, Philos. Mag. 12, 573 (1965).

[44] C. Baker and A. Kelly, Philos. Mag. 11, 729 (1965).

[45] J. Kotakoski, A. V. Krasheninnikov, U. Kaiser, and J. C. Meyer, Phys. Rev. Lett. 106, 105505 (2011).

[46] L. Liu, J. Gao, X. Zhang, T. Yan, and F. Ding, Nanoscale 6, 5729 (2014). 